\title{
A functional and structural diagnosis of online health communities sustainability: A focus on resource richness and site design features
}

Hyang-Sook Kim ${ }^{\mathrm{a}}$, Amy Mrotek ${ }^{\mathrm{b}}$

a Department of Mass Communication \& Communication Studies, Towson University, 132 Stephens Annex, 8000 York Road, Towson, MD 21252, USA

b St. Norbert College, De Pere, WI 54115, USA

\begin{abstract}
The reality of online communities' under-contribution issues has often been clouded with theoretical rather than real-world insight. The present study aims to neutralize this disparity, through content analysis on 196 health websites and online communities to systematically evaluate their functional and structural interfaces - the ingredients for a thriving online environment. Particular attention is paid to what variables equate to successful site traffic and impressions, ultimately providing suggestions to facilitate and optimize user contribution. While the majority of health websites and online health communities offered users fairly rich information about general health concerns, user environments in online health communities significantly lacked both structural and functional cues to encourage user contribution. External sponsorship could mitigate the discrepancy between the real world situations and academic suggestions.
\end{abstract}

Keywords:

Online community sustainability, Online health community environment, Resource-availability approach, Interface-structure approach, Website content analysis

\section{Introduction}

Previous literature has widely investigated the problem of under-contribution in knowledge sharing and social support domains (e.g., Kim \& Sundar, 2014; Nonnecke, Preece, \& Andrews, 2004; Preece, Nonnecke, \& Andrews, 2004; Voelpel, Eckhoff, \& Förster, 2012). Beginning with classifications on posters/contributors, lurkers, and latent users, otherwise known as newcomers, the identified literature primarily reasons that people tend to expect more resources in these online settings than they are willing to offer to themselves (Fox \& Jones, 2009; Preece et al., 2004). From this, studies continued to examine the potentiality of technological affordances that could help reduce lurking populations in online communities and foster burgeoning contributions (e.g., Farzan, Dabbish, Kraut, \& Postmes, 2011; Kim \& Sundar, 2014; Lampe, Wash, Velasquez, \& Ozkaya, 2010; Ling et al., 2005; Ridings \& Gefen, 2004). Such a line of research provides greater theoretical implications and practical applications for the environment of healthrelated knowledge and support sharing than other domains of knowledge sharing for which most of people willingly devote their time, genuinely driven by their personal-health interest, experience, and expertise (e.g., Fox \& Jones, 2009; Lampe et al., 2010). 
In fact, researchers have focused on this problem of under-contribution because it has emerged as such a significant node in the formation of online community sites, particularly when considering their sustainability (Bishop, 2007; Butler, 2001; Nonnecke \& Preece, 1999; Preece \& Maloney-Krichmar, 2005; Preece, 2000, 2001). The chief principle of these online communities' operation and growth is to generate resources for community users, encouraging users to continue their membership within that site (Valacich, Dennis, \& Nunamaker, 1992; Wasko \& Faraj, 2000). As a result, the communities expect to grow in size. On one hand, the critical mass theorists (e.g., Oliver \& Marwell, 1988; 2001) argue the production of available resources in online communities rely on a critical but small group. This group makes up only a fraction of the whole community yet plays a chief role in its sustainability, as they serve as all-the-time contributors based on their own expertise and interests, regardless of a presence of external reinforcements such as interface-supported community environments or continuous interactions with other users (Preece et al., 2004). However, this assumption of the critical mass theory may be dangerous to the life cycle of online "health" communities in that information, voices, and advice from a limited number - although still influential to other community members - could impede the diversity of the sites (e.g., Cline \& Haynes, 2001).

Given the significance of unbiased, thriving online health communities as a result of continuous contributions by a wide range of user groups, previous literature has addressed key factors that would effectively deter lurking tendencies (e.g., Bishop, 2007; Chidambaram \& Tung, 2005; Farzan et al., 2011; Kim \& Sundar, 2011; Ling et al., 2005). What is missing from this body of literature, however, is a comprehensive diagnosis on the real environment of these online health communities with respect to their functional and structural adequacy, as well as the likelihood for the sites' success and sustainability. Do online health community sites offer users a viable environment to facilitate and optimize user contributions? Thus, the present study attempts to systematically assess the status quo of user environments in online health communities. Drawing primarily from online lurking and community sustainability (e.g., Butler, 2001; Preece et al., 2004), social support (e.g., Cutrona, 1990), and user perceptions on technological affordances research (e.g., Kim \& Sundar, 2011), the present study conducted a content analysis with 196 health websites and online communities, offering online health community sites practical insights for their long-term success in addition to suggesting theoretical approaches to curb under-contribution for health communication research.

\section{Literature review}

\section{Mechanisms of online community operation and sustainability}

Thanks to new media technology, online health communities now gather users based on their common interests and needs without geographical limitations, further encouraging members to share their knowledge and experiences with other members (Kollock \& Smith, 1999; Ridings, Gefen, \& Arinze, 2002). In order to maintain the ideal cycle of online community operation by fulfilling this fundamental definition, online health community sites need a sustainable environment for both resource generation and size expansion (Faraj, Jarvenpaa, \& Majchrzak, 2011).

\section{Requirements for online community operation, user contributions and sustainability (or lack thereof)}

Online communities are similar to the basic concept of a real-world, physical community insofar as people visit them, do their business generally obtaining necessary information and support - and leave with or without having the intent to revisit (Faraj et al., 2011). Therefore, one of the critical factors in online communities is having sufficient resources for people to create and/or consume. Of course, the resources exchanged through online communities, as a form of public goods, are free (Kollock, 1999). This resource- 
based point of view calls for other subsequent factors with regard to the structure and function of online communities. For instance, users do not have particular obligations to create public goods even if they do have the need to consume it. Few individuals are willing to pay more than what they can gain from a pool of public goods. As a result, no one is likely to participate in sharing information if $s /$ he knows others did not put much effort into the sharing process. Such a user perception, in turn, leads to an "undersupply of discretionary information" (Connolly \& Thorn, 1990, p. 221; Fox \& Jones, 2009). In order to prevent information undersupply, online communities focus on expanding community size by recruiting more members (Mo \& Coulson, 2010).

In order to attract more community members, online communities need an effective user environment in which members are willing to not only spend time with the site but also share their experiences with others. The importance of interface design now comes into play, attracting users to go beyond being newcomers or lurkers. Latent users who are at a monitoring stage as well, seeking community membership and quietly preparing for future contribution, would show selective and consistent motivation to participate once they consider contributing to an appealing community (Valasquez et al., 2014). To appease all these kinds of potential community members - from newcomers to latent members - online communities need to present (1) how relevant their communities are to users, (2) how diverse their members are, and (3) how efficient their community interface is for the members' knowledge and support sharing (Toral, Martinez-Torres, Barrero, \& Cortes, 2009). As a result, such members would perceive benefits from the community, negating hesitancies on the cost of contribution (e.g., time to spend with the site and sharing activities) (Li, 2011).

Needless to say, a well-designed online community is the priority for online community success and sustainability. A critical point needs to be noted, however: People are mostly cognitive misers, quickly scanning sites to glean what is offered in terms of site features, resources, and community size, rather than carefully examining the site resources and structure for a long period of time (Chaiken, 1987; Kim \& Sundar, 2011; Petty \& Cacioppo, 1986). Thus, online communities need to impress site users - current and prospective - with a variety of cues instantly translating the cohesiveness, diversity and efficiency of their community sites (Toral et al., 2009).

\section{Functional interface cues for resource richness}

\section{Community size, health topics, and information breadth and depth}

Community culture along with resource availability is not easily detected unless each member spends a certain amount of time with the community. In this case, most members want to appraise community structure and resource with a quick scan. Based on such cues (e.g., community size information, topical interest of community members, a number of menu tabs for information depth and breadth), newcomers could form perceptions on the overall community function and value, ascertaining the viability of joining and hence making their decision to stay with the community or leave (Chu, 2009).

Ren and Kraut (2014) also found a wide breadth of topics and great depth of information positively influenced online community users' commitment. In addition, user contribution increased when the community site offered an interface feature that allowed user-controlled content moderation. Between the two types of content moderation features, community-level moderation - a common interface feature that filters out appropriate or relevant information to a user across a macro site level - only predicted user commitment given a narrow topic breadth. On the other hand, when the study simulated a personalizedlevel moderation - a user-controlling content filtering system (e.g., topic selection drop-down menu or user-to-user matching system on a user profile page) - it predicted user commitment and contribution given a wide breadth of topics and in-depth information.

R1. Do online health community sites well display cues that signal community resources regarding (a) community size, (b) focal health topics, (c) depth and breadth of information and support and (d) topic 
filtering system and content moderation for users?

\section{Support systems and types of support}

Given the positive relationship between user satisfaction with online communities and online community success (e.g., Stoerger, 2007; Wang \& Clay, 2012; Zhang \& Yang, 2009), optimal matching theory (hereafter OMT, Cutrona, 1990) suggests satisfaction amongst site users will be determined by the degree to which the users find their needs adequately addressed and served. Therefore, it is critical to anticipate community members' satisfaction with a balanced, rich pool of social support, ideally with one or multiple site features geared toward need-matching (e.g., Turner, Grube, \& Meyers, 2001; Wright \& Bell, 2003; Wright, Johnson, Bernard, \& Averbeck, 2011). OMT classified five types of social support: "emotional support (expressions of comfort and caring), social integration or network support (membership in a group where members share common interests and concerns), esteem support (bolstering of the person's sense of competency or self-esteem), tangible support (services or resources) and information support (advice or guidance)" (Cutrona, 1990, p. 7).

Previous studies confirmed a mixture of these five social supports would benefit community members addressing health matters. A content analysis of 755 actual posts found in a social support group for survivors of sexual violence showed that the community members of this online group sought emotional support (42.6\%), information (21.2\%), esteem (20.9\%) and network support (13.1\%) (Yeager, 2011). Online communities for rare diseases also showed a variety of social support needs, not only in esteem building but likewise with heavy amounts of informational aid (Chen, 2012; Coulson, Buchanan, \& Aubeeluck, 2007). Online health communities considering a wide range of health topics and concerns need to assess their support channels, noting a rich pool of social support as a requirement for success and sustainability.

R2. Do online health communities offer users five types of social support as OMT suggests?

\section{Structural cues for interface design}

Preece (2001) articulated the importance of structural aspects for viable social interaction in online communities. Technological affordances aid in this process, with content-structural requirements for successful online communities classified into four dimensions: interactivity (e.g., interface features for easy reply, content evaluation, easy entry to new posts as they serve as the technological indication for reciprocity between users), navigability, accessibility, and aesthetics (Preece, 2001). Furthermore, the professional presentation of a website influenced site users' overall positive impressions. Interface design features should be key to this impression as well. Content-related site attributes, such as the quality of information marked by expertise and trustworthiness labels, operate as heuristic cues for users' perceptions, ultimately guiding their decision-making process as a more positive and interactive one (Fogg et al., 2001).

\section{Interactivity}

Interactivity is conceptualized - among many approaches - in three distinct dimensions: medium interactivity, source interactivity, and message interactivity. These three dimensions can be characterized as site-centered, user-centered, and user-to-site/ user-to-user centered, respectively, depending on the focus of the interactivity with its technological affordances (i.e., what interface features does the user have access to) as well as operational outcomes through the technological affordances (i.e., deliverables of tasks performed given the affordances and psychological influence of the interactions that occur in the interface) (Sundar, 2008) ${ }^{1}$. In online health communities, source and message interactivity appear significant and are

\footnotetext{
${ }^{1}$ Medium interactivity is excluded from this discussion because of its lack of ecological validity. Typical technological affordances for the medium interactivity such as tools for zoom-in and out, mouse-over, sliding bar, cover-flow, etc. (Sundar et al., 2014) are rarely found in online health communities.
} 
commonly embedded interface features (e.g., membership history/expertise, reply, star ratings or likes, social plugins, one-click posting with or without login, etc.). Previous studies found these interface cues significantly affect and even readily signal the possibility of user-initiated actions (i.e., user contribution by posting in user profile, replying and/or forwarding information from a community to external sites) and user-to-site/ user-to-user contingent message exchanges (e.g., search history, live chat, buddy system, etc.), contributing toward a positive impression on the sites (Sundar et al., 2012) and users' intention for future contribution (Berlanga, Rusman, Bitter-Rijpkema, \& Sloep, 2009; Kim \& Sundar, 2011).

Even earlier, Heeter (1989) proposed six aspects of website interface design to help estimate the levels of site interactivity with features that allow users (1) to choose information from comprehensive and sufficient pool of resources, (2) to demonstrate their contributions to the site or observe other users' contributions, (3) respond to other users promptly or vice versa, (4) to confirm the site's moderating role to regulate information as well as users' activities in the site, (5) to contribute with ease, and (6) to foster user- to-user interactions. Furthermore, in order to create a strongly-bonded community culture, online communities need to secure interface design features with which community users can (1) easily find the information and resources they need, (2) develop affection with other users and (3) form community commitment (Bateman, Gray, \& Butler, 2011). Likewise, interface features that encourage user actions are considered to be critical to user contribution. In particular, users appreciate their community acknowledging their activities. A sheer presence of community feedback and praise potentially prompts user contribution, and hence secures community success and sustainability (Berlanga et al., 2009; Zajonc, 1965; Zajonc \& Brickman, 1969).

In particular, site's user-centered and user-to-user interactivity features have been found to be effective for encouraging contribution. A survey of 632 members of an online community showed that structural support systems, including contribution recognition features (e.g., content evaluating tools such as star ratings or likes), combined with perceived community value and an absence of contribution obligation predicted user commitment and participation (Kang, Lee, Lee, \& Choi, 2007). Similar interface features that primarily foster a culture of community feedback satisfies the principle of reciprocity for community users, fulfilling their sense of membership. In addition, acknowledgement of user contribution has a positive impact on further involvement. Users' satisfaction and thereby intent for knowledge sharing has been increased not only by their contribution and self-efficacy, but also by reciprocal relationships among users (Cheung, Lee, \& Lee, 2013). Another study also found a widely used reputation system for online knowledgesharing communities (i.e., Karma for Slashdot) operated as a boost for user contribution (Ganley \& Lampe, 2009).

With respect to the user-to-user interaction, the previous literature proposed the tendency for individuals to avoid social responsibility when the existence of others is apparently visible (Karau \& Williams, 1995; Latane 1981). On the contrary, a site feature that helps reduce this phenomenon of social-responsibility dilution might see an increase in user contribution as long as the feature is offered through user's choice (Kim \& Sundar, 2014). Buddy systems offer a tangible solution for this reduction of a bystander effect (Du, 2006; Kim \& Sundar, 2014), providing one such interface cue that signals ease of social interaction. This, in turn, will increase positive impressions and higher user contribution (Preece, 2001).

In fact, a content analysis of 30 health websites dedicated to palliative care conducted by Banna, Hasan, and Meloche (2009) found that sites have improved website functionality when users search and access information they need as well as contribute their own. However, various features that might facilitate user-to-user interaction (e.g., user profile, live chat rooms, feedback cues on discussion forums, etc.) were missing. There was little option for personalization/customization, multimedia inclusion, and content immediacy in the sites analyzed for the study.

R3. Do online health communities deploy essential interface cues that signal sufficiency of site interactivity? 


\section{Navigability, accessibility and aesthetics}

Structural requirements also include pleasantly displayed information (i.e., aesthetic state of online communities), easy site navigation, and readily accessible information (Preece, 2001). Thielsch, Blothenberg, and Jaron (2013) examined three dimensions of functional and structural website features content, usability, and aesthetics - to estimate user impression and evaluation on the sites. A series of studies using both user surveys and content analysis confirmed that aesthetic aspects as well as usability are critical to predict a user's impression and subsequent evaluation before becoming too familiar with the sites. Usability "is a quality attribute that assesses how easy user interfaces are to use" (Nielsen, 2003). Features that help users complete their tasks without hassles (i.e., ease of use and ease of accessibility to information) (Flavián, Guinalíu, \& Gurrea, 2006) are essential to attract site users at first glance. Phang, Kankanhalli, and Sabherwal (2009) further found that easy system use was critical to user contribution. Given the scarcity of related research in the context of online health communities, it should be interesting to assess their interfaces' usability (navigability and accessibility) and aesthetics, as these cues signal site professionalism, often influencing user impressions as well as contribution (Fogg et al., 2001).

R4. How professional does the interface of online health communities look based on its navigability, accessibility and aesthetic state?

\section{Privacy protection and external funding}

As perceived credibility shows a strong positive relationship with a user's overall site impression (Hong, 2006), two key factors - privacy protection and externally funded sources - also need to be assessed in the present study. Health information often carries the expectation of being personal and private (e.g., Agaku, Adisa, AyoYusuf, \& Connolly, 2014). Thus, the presence of interface cues that signal user privacy or identity protection is critical to user impression of the health site. Contrary to the negative stereotype between age and online community participation, a study found the key for online community features lies in privacy protection and a rich amount of resources encouraging user contribution, rather than users' ability to adapt to the interface's technology for information sharing (Chung, Park, Wang, Fulk, \& McLaughlin, 2010). In other words, site users would exhibit a strong willingness to contribute when they develop trust with the site, regardless of age or ability to adopt its technological features.

R5. Do online health communities offer an interface cue that signals privacy protection for users?

Lastly, users of online health communities are normally concerned about information credibility when the information is possibly commercialized due to externally funded sources, coming in the form of outside advertisements and sponsorship (Flanagin \& Metzger, 2000; Hong, 2006). A content analysis of 60 healthrelated websites found the number of interactive features and the level of credibility were negatively correlated when funding sources were present (Stoerger, 2007). However, another study suggested that user-friendly design features, in-depth content availability, and sophisticated site interfaces appeared to be more critical to site users' perceived credibility rather than a presence of sponsorship. In other words, when

such sponsorship existed, users would appraise the quality of content and interface design features of the site prior to assessing site credibility (Flanagin \& Metzger, 2007).

R6. What is the relationship between presence of (a) external advertisements and (b) sponsorship and structural quality of online health communities?

\section{Method}

The present study conducted a content analysis of 196 online health websites and communities. Originally, a total of 221 websites was located based on the results from the top search site for online support groups, under 
the category of Health, based on website traffic estimates (Alexa.com, 2014). Twenty-six sites were excluded from the original sample list due to certain disqualifications: a cease of that site domain, site errors, repeated sites with other health topics under the identical domain name (e.g., Healthboards.com), or non-English sites. Two independent coders completed the initial coder training for inter-coder reliability with 43 sites (about $20 \%$ of the total samples in the initial list) and finished the inter-coder reliability stage after two more rounds of coder-training sessions to reach satisfactory levels of reliability, ranged from 0.70 to 0.99 on Krippendorff's alpha for both nominal and interval variables coded for the study (see Table A1 for the complete inter-coder reliability). The unit of analysis is a website.

\section{Coded variables}

In order to confirm whether a site had an actual online community or not, two levels of community existence were coded. The presence or absence of online community was determined as places where users could share thoughts, emotions, information, stories, etc., such as message boards/forums/chat rooms or similar online communication interface features (Kim \& Sundar, 2014). Community size was operationalized as the volume of the site traffic. Coders located the information cue for how many people visited a website (e.g., the number of site visitors, members of the site, etc.), which was a ratio variable. Focal health topics are defined as the most dominant health issue discussed in a coded site. Initially, this variable was coded as an open-ended variable. After the completion of the coding, a seven-category list of focal health topics (i.e., cancer, mental health including sleep disorders, mental illnesses and depression, disability, rare diseases, smoking, general health concerns, and other) was created. This variable, then, was recoded into two focal health issues - chronic, acute and/ or high-maintenance (cancer, mental health, disability, rare diseases) vs. general and broad (smoking, general health concerns, other).

Three separate site category variables were coded. First, depending on the neutrality of the interest of a site, a website can be coded as either health-focused (i.e., direct interest) or general (i.e., indirect interest), which included health topics at some level (Flanagin \& Metzger, 2007, p. 325). Topic breadth and depth were coded as independent, two-ordinal variables (Chu, 2009; Ren \& Kraut, 2014). The topic breadth variables coded broke into three different levels: narrow, medium and wide. The coding principles for the topic breadth were as follows. First, if a site contained a seemingly wide range or well-rounded amount of categories, it would fall into wide breadth. Even if that range were all dedicated to the same or similar health topic, if there is a large amount of categories present, it would be categorized wide. The converse situation qualifies the health community as narrow, while the situation between wide and narrow would fall under medium. The topic depth was also coded into three levels, shallow, medium and deep. The depth of an online health resource/community included any information found directly on their domain and information that linked to other pages. Even if those links redirected the user to an external domain or different website all together, it would still qualify that original website as deep versus medium or shallow. For example, if a site addressed general men's health, the site would be considered a wide breadth of topics but shallow in depth if the amount of information gained directly or indirectly from linked external sites was not rich enough for users. If multiple sclerosis was a core topic discussed in a female pregnancy website, then the site would be coded as a narrow breadth but deep in depth.

Topicfilteringsystem is defined as an interface feature allowing site users to readily locate a health/discussion topic within the site. Topic filtering interfaces include, but are not limited to, features such as a typeable search bar and topic headers that, once clicked, will redirect the user to a different page with a specific, named topic. Content moderation was coded "yes" (vs. no) if an interface feature indicated whether the site monitors content posted. Specifically, a content moderator could be defined as another human being whose purpose was explicitly stated to be a website administrator or forum moderator. Content moderators additionally extend to website features, such as having to apply for membership before fully accessing the website or a "Report this post/member" function (which makes you yourself a website monitor). Information currency was coded using a single item, "the information posted in the site is current," (1 being strongly disagree to 7 being strongly agree) based on the date of the last update of the site and the number 
of posts in the last week.

The type of social support variable was defined as the primary purpose of the online community or of the site itself based on OMT (Cutrona, 1990, p. 7). Informational support included message boards/forums/chat rooms/webpages which focused on advice and guidance for site users. Tangible aid was chosen for the variable if message boards/forums/chat rooms focused on services and resources that site users could apply or utilize in their real-world, daily setting. Emotional support was coded when message boards/forums/chat rooms focused on site users' expressions of comfort, care and empathy. Social network support was coded when message boards/forums/chat rooms focused on site users' membership maintenance and membership orientation in a group/ site where site users could obtain/share common interests and concerns. Lastly, esteem support was coded when message boards/ forums/chat rooms focused on site users' sense of competency, self- esteem, self-image or the like. When message boards/forums/chat rooms combined more than one type of support, then the site was coded as a combination category. For example, can emotional support lead to tangible aid in the same forum, where members start contributing resource suggestions (e.g. suggesting a renowned doctor or relevant health book to comfort a fellow user)? An "other" category was also added to make the variable categories exhaustive. ${ }^{2}$

User profile was operationalized as a webpage in which a user could create a personal page with basic or in-depth information about him/herself. Membership history was coded "yes" (vs. no) if an interface feature informed on a site user's years of membership. Member expertise was coded "yes" (vs. no) if an interface feature deemed a site user's commitment to the site/community, rank, position and/or alike. Member contribution history was coded "yes" (vs. no) if an interface feature indicated the number of posts a site user contributed. Reply function was coded "yes" (vs. no) if an interface feature allowed a site user to leave a message or reply to other users' questions/posts/etc. Content evaluation was coded "yes" (vs. no) if an interface feature indicated popularity of posts or content such as the number of replies, likes and/or shares, star ratings, and/or alike. Content sharing was coded "yes" (vs. no) if an interface feature allowed a site user to share posts/content of the site. This variable also included, but was not limited to, features which allowed posting and sharing the website's content directly to social media pages such as Facebook, Twitter, and other social media outlets (Kim \& Sundar, 2011). Buddy system was coded "yes (vs. no) if an interface feature paired up users (more than one user) to initiate interactions (Kim \& Sundar, 2011). This included both online connection through email and other means of electronic communication and/or through traditional communication modes such as mail and telephone. ${ }^{3}$

Navigability was defined as the degree to which a user can transport from one page to another in a site (Herndon, van Dam, \& Gleicher, 1994; Jul, 2004; Sundar, 2008). A five-item scale (a 0.88) was used to assess site navigability (e.g., "It does not take a long time to navigate through the website to find something") (1 being strongly disagree to 7 being strongly agree; Jin, Park, \& Kim, 2010; Preece, 2001). For the aesthetic state of a site, the present study used a shorter version of the web interface aesthetic scales modified by Moshagen and Thielsch (2013). Four dimensions of an aesthetic web interface assessment are constructed in this scale: simplicity, diversity, colorfulness and craftsmanship. Example items from each dimension are "the layout is easy to grasp," "the layout is pleasantly varied," "the color composition is attractive," and "the layout appears professionally designed." The complete coded data resulted in a satisfactory level of inter-item reliability, a 0.96 .

\footnotetext{
2 After the completion of the first round of coding, the coders found that most websites were encompassing, and possessed features qualifying them as "combination category." Initial inter-coder reliability disparities were attributed to this nature of the types of social support each site offered. Therefore, the coders focused on the support types most explicitly found rather than on the first step of categorizing it as a combination.

${ }^{3}$ Live-chat function was not reported in the study due to a significant low number of sites that featured this interactivity function $(n 1 / 416)$.
} 
Accessibility was assessed by convenience, readiness, responsiveness, and availability of site resources using a four-item scale ( 1 being strongly disagree to 7 being strongly agree; Jin et al., 2010; Preece, 2001). Example items are "Website is open to anyone, anywhere, at any time," and "Users download the software components and run them in reasonable time" $(\alpha=0.69)$. Login requirement was coded "yes" (vs. no) if a site required a user to log into the site in order to view/read relevant information or share their experiences. For the posting entry step, coders first checked the number of steps (i.e., webpage layers) necessary to post on the site, with the website left blank if no posting feature existed. Second, coders assessed the degree of complexity to posting entry ( 1 being not at all complex to 7 being extremely complex). After initial coding, coders broke down the numbers of posting-entry steps to code the second scale measure as follows: 1 step being coded as "1"; 2 e3 steps being coded as "2"; 4 steps being coded as "4"; 5e10 steps being coded as "6" and more than 10 steps being coded as "7." Privacy protection was coded "yes" (vs. no) if an interface feature signaled a site would be sensitive to user privacy. Specifically, if a site contained a page/pages, notifications, or messages of any kind that explicitly articulated the website's "privacy policy" or "user privacy promise," then the site was coded as a "yes." If no such page/notification/message was to be found, then it was coded as "no." Lastly, a presence of external funding sources was coded by two separate variables: presence vs. absence of advertisements and sponsors. Sponsorship refers mainly to the existence of "donate," "sponsor," or "support" tabs or features on an online health community. It also included any attempts from the organization or charity to elicit monetary funding from website users.

\section{Results}

\section{Relationships between online health communities and functional cues for resource richness}

Of the 196 coded websites, $95 \%$ of the sites were truly health focused. Almost half the total websites coded $(49 \%)$ offered users a kind of online community space $(\mathrm{N}=196)$. Only 34 community sites indicated its size, the average size of which was 83,864 ( $S D=233,383$, Minimum: 30; Maximum: 1,086,704) [R1(a)]. Regarding focal health topics [R1(b)], $60 \%$ of the sites that offered a form of online community to users focused on chronic, acute, and/ or high-maintenance health concerns. With this comparison between the presence and absence of an online community, the focal health concerns featured slightly different percentage patterns, such that $9 \%$ more chronic, acute, and/or high-maintenance health concerns were mainly discussed in health websites without an online community than with an online community. However, general and broad health concerns were more popular in sites with online communities than without them, $\chi_{(1,190)}^{2}=4.93, p<0.05$ (Fig. 1).

Regardless of the existence of an online community, more sites covered a wide range of topics (46\%) than medium (32\%) and narrow (22\%) ranges (Fig. 2). Similarly, more sites, regardless of presence or absence of an online community, presented in-depth information (53\%) than medium (30\%) and shallow (17\%) (Fig. 2) [R1(c)]. The number of sites to feature some form of topic-filtering systems for users did not differ between presence and absence of online community (Fig. 3).

However, content moderation differed with the presence and absence of an online community, $\chi^{2}(1,192)$ $17.41, p<0.001$ such that more sites with an online community contained a content moderation function $(20 \%)$ than those without a community $(7 \%)$, whereas typically sites without an online community did not include any content moderation function (44\%) compared to those with an online community (30\%) (Fig. 4). For the information currency variable, sites with an online community appeared to be more current $\left(M_{\text {with }}\right.$ online community $=4.84, S D_{\text {with }}$ online community $\left.=2.00\right)$ in terms of their information updates than those without an online community $\left(M_{\text {with online community }}=4.22, S D_{\text {with }}\right.$ online community $\left.=1.79\right), t(188)$ 
$=2.25, p<0.05[\mathrm{R} 1(\mathrm{~d})]$.

Regarding social support, the coded sites offered users (R2), esteem support first and foremost (59\%), followed by tangible aid (25.5\%), instrumental support $(6.3 \%)$, emotional support $(3.6 \%)$, and then a combination of either these five social support categories. When these percentages are broken down to factor in the presence or absence of an online community, tangible support was more available in the sites without an online community $(88 \%)$ than with it $(12 \%)(n=49)$, whereas esteem support was most available in sites with online communities $(67.5 \%)$ than without $(32.5 \%)(n=114)$.

\section{Relationships between online health communities and structural cues for interface design}

Among the 95 sites with online communities, basic interface features for both source and message interactivity were very limited, most commonly displaying membership history (20\%) and a member's contribution history (28\%), membership expertise (16\%), content evaluation (11\%), reply function $(60 \%)$, and content sharing (48\%). Only $10 \%$ of sites with online communities offered users a form of an online buddy system $(n=95)(\mathrm{R} 3)$. Navigability $\left(M_{\text {with }}\right.$ online community $=4.76, S D_{\text {with }}$ online community $=0.86$; $M_{\text {without }}$ online community $=4.92, S D_{\text {without }}$ online community $\left.=1.00\right)$ and aesthetics $\left(M_{\text {with }}\right.$ online community $=4.42, S D_{\text {with }}$ online community $=0.92 ; M_{\text {without }}$ online community $=4.55, S D_{\text {without }}$ online community $=$ $1.14)$ between the sites with $(n=93)$ or without online community $(n=97)$ did not statistically differ. However, accessibility was slightly better in the sites without an online community $\left(M_{\text {without online community }}=\right.$ $5.57, S D_{\text {without }}$ online community $\left.=0.82\right)$ than those with one $\left(M_{\text {with }}\right.$ online community $=5.36, S D_{\text {with }}$ online community $=0.78), t(188)=1.82, p=0.07$. On a 7-point scale, the average posting entry steps was 3.95 , with 1.52 of $\mathrm{SD}(n=55)(\mathrm{R} 4)$.

Seventy-two percent of the sites coded did not offer users any type of privacy protection features $(\mathrm{N}=$ 192). When this percentage was broken down between the sites with and without online community, the sites with online communities had a greater number of privacy protection features than those without any. This pattern was reversed for the absence of privacy protection $\left(\chi^{2}(1,192)=5.46, p<0.05\right.$, Fig. 5 (R5).

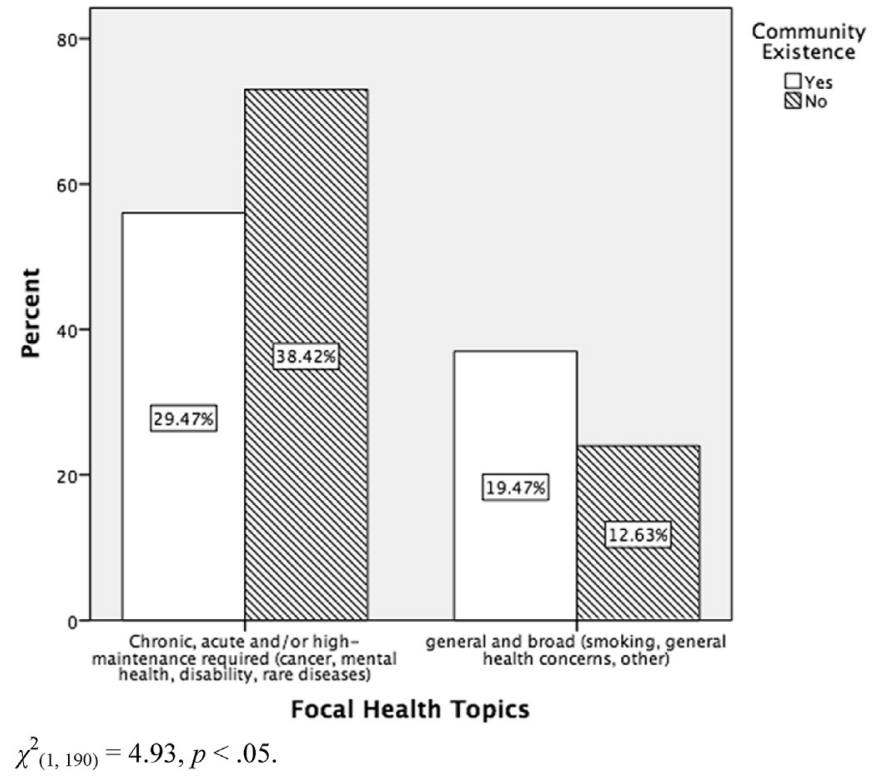

Fig. 1. Association between community existence and focal health topics. $\chi^{2}(1,190)=4.93, p<0.05$. 

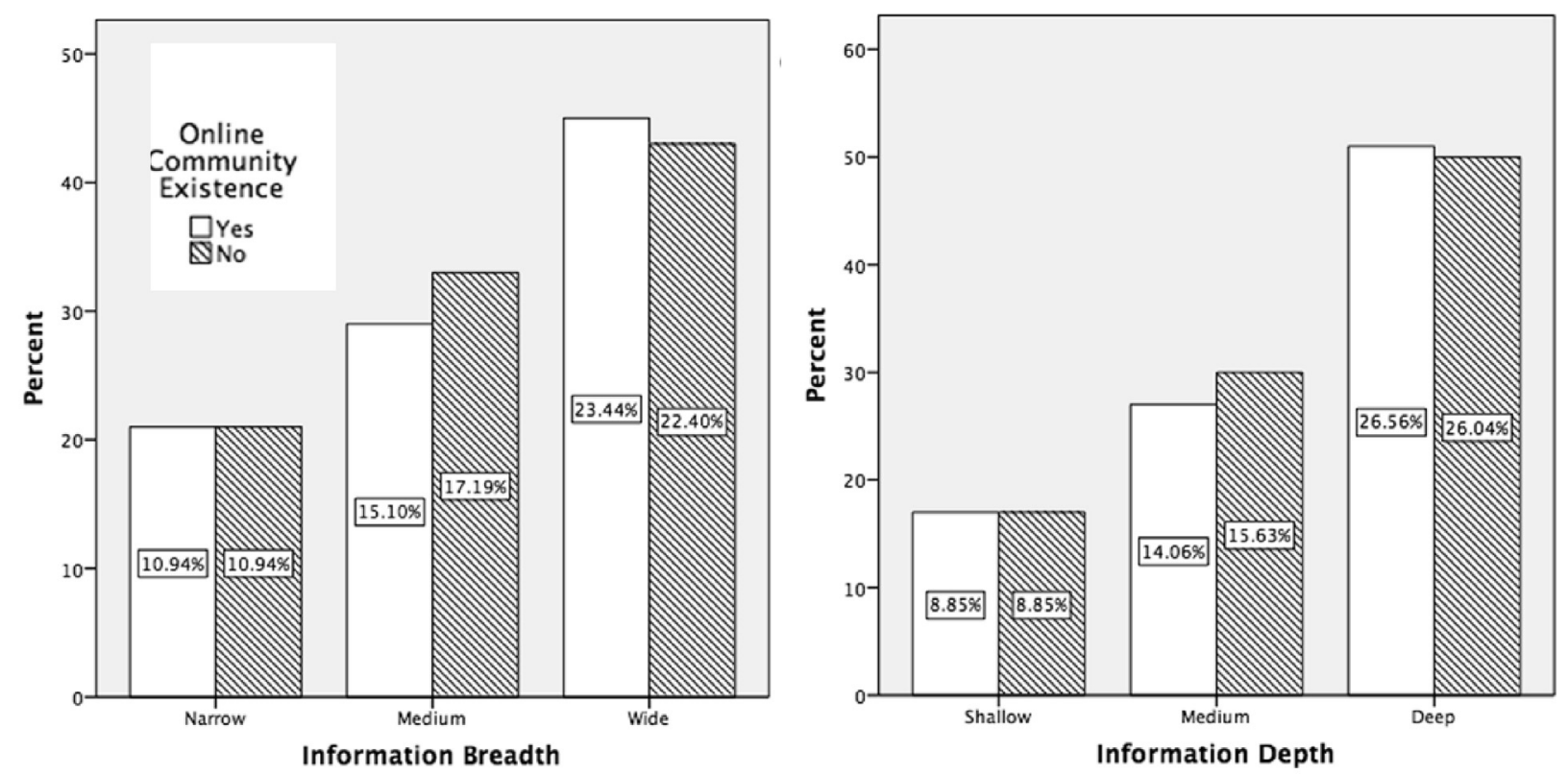

$\chi_{(2,192)}^{2}=.28, p>.05$ (left) $1 ; \chi_{(2,192)}^{2}=.15, p>.05$ (right)

Fig. 2. Association between community existence and information breadth (top) and depth (bottom). $\chi^{2}$ (2, ${ }_{192)}=0.28, p>0.05$ (left) $1 ; \chi^{2}{ }_{(2,192)}=0.15, p>0.05$ (right).

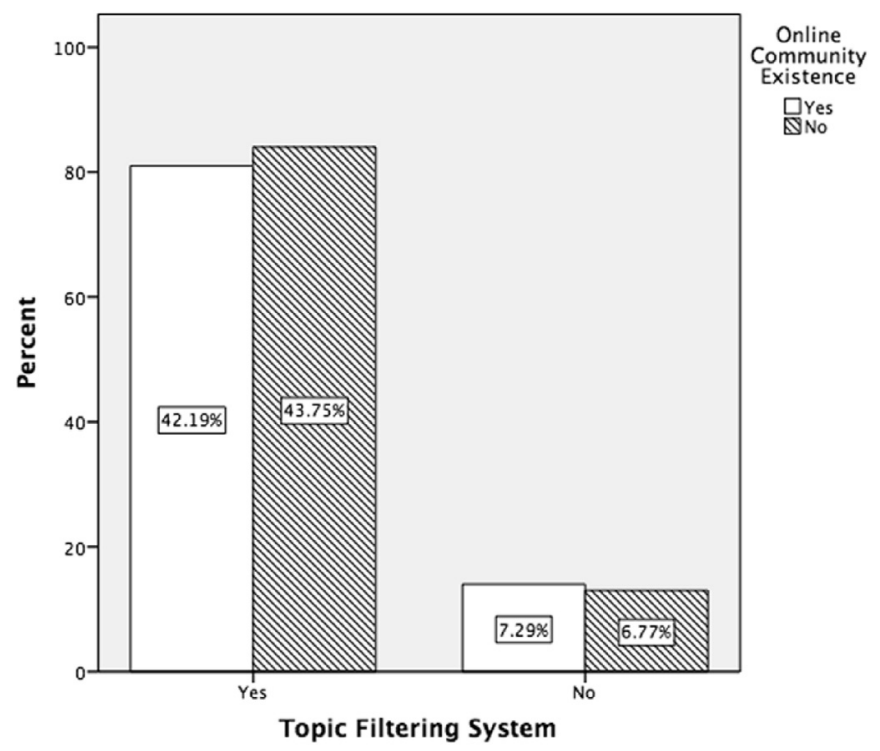

$\chi^{2}(1,192)=.07, p>.05$.

Fig. 3. Association between community existence and topic filtering system. $\chi_{(1,192)}^{2} 0.07, p>0.05$. 


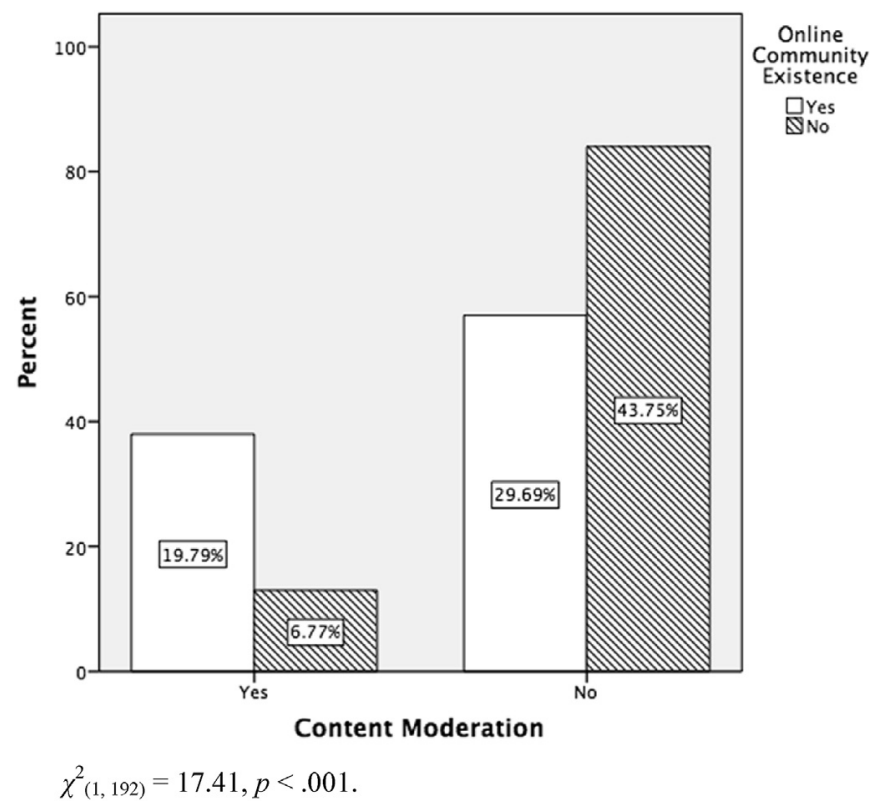

Fig. 4. Association between community existence and content moderation. $\chi_{(1,192)}^{2}=17.41, p<0.001$.

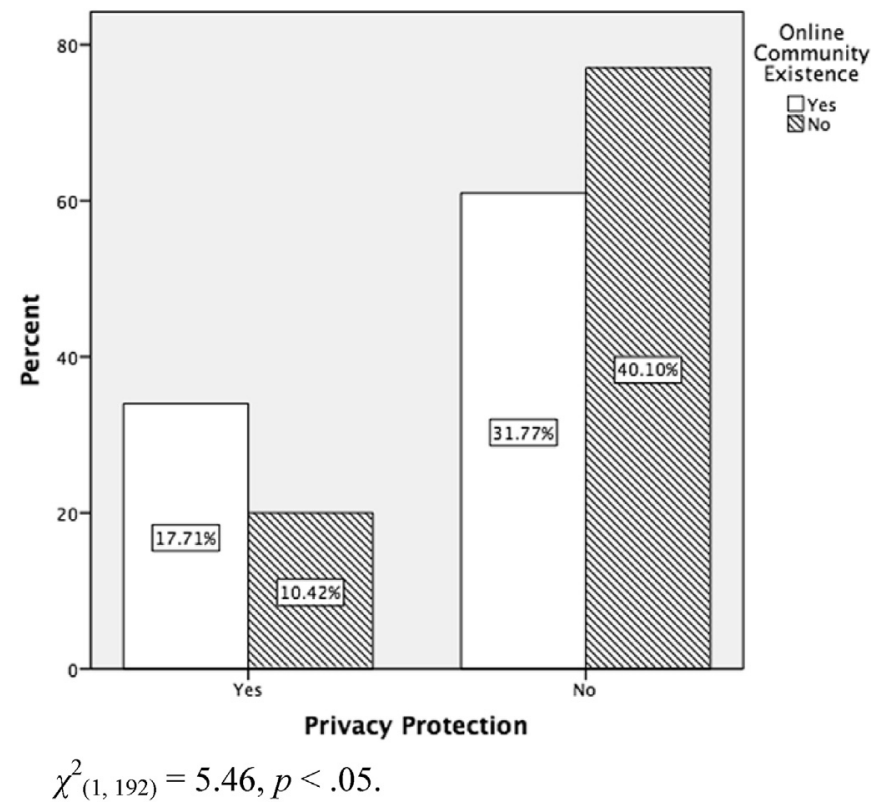

Fig. 5. Association between community existence and privacy protection. $\chi_{(1,192)}^{2}=5.46, p<0.05$.

Overall, advertisements were not consistently present in the coded sites, but became more prevalent in sites without online communities ( $83 \%$ vs. $17 \%$ of the sites that included external advertisements) than those with them ( $58 \%$ vs. $42 \%$ of the sites that included external advertisements), $\chi^{2}{ }_{(1,192)}=13.89, p<0.001$. On the other hand, both the sites with $(62 \%$ vs. $38 \%$ of the sites with external sponsorship) or without online communities (54\% vs. $46 \%$ of the sites with external sponsorship) did not have or indicate external sponsorship, $\chi_{(1,192)}^{2}=1.42, p>0.05$. Regarding the structural quality of the sites coded, depending on the presence (vs. absence) of external funding sources, a series of a $2 \times 2$ factorial variance analysis was performed. Analyses found the main effect of advertisements on site accessibility, $F(1,186)=10.02, p<0.01$ to be that sites without external advertisements appeared to be more accessible to information $(M=6.00, S D=0.73)$ than those with external advertisements $(M=5.16, S D=0.89)$. Main effects of external sponsorship were found 
on navigability $[F(1,186)=6.32, p<0.05]$ and aesthetic states, as well $[F(1,186)=24.64, p<0.001]$.The sites with external sponsorship were easier to navigate $(M=5.05, S D=0.78)$ and looked more visually appealing $(M=4.91, S D=0.90)$ than those without sponsorship $(M=4.69, S D=1.02$ for navigability; $M=4.18, S D=$ 1.04 for aesthetic state). In addition, a marginally significant interaction effect was found between the online community existence and external sponsorship, $F(1,186)=3.46, p=0.05$. This external sponsorship appeared to be more important to the aesthetic state of sites without online communities than those with online communities, as shown in Fig. 6 (R6).

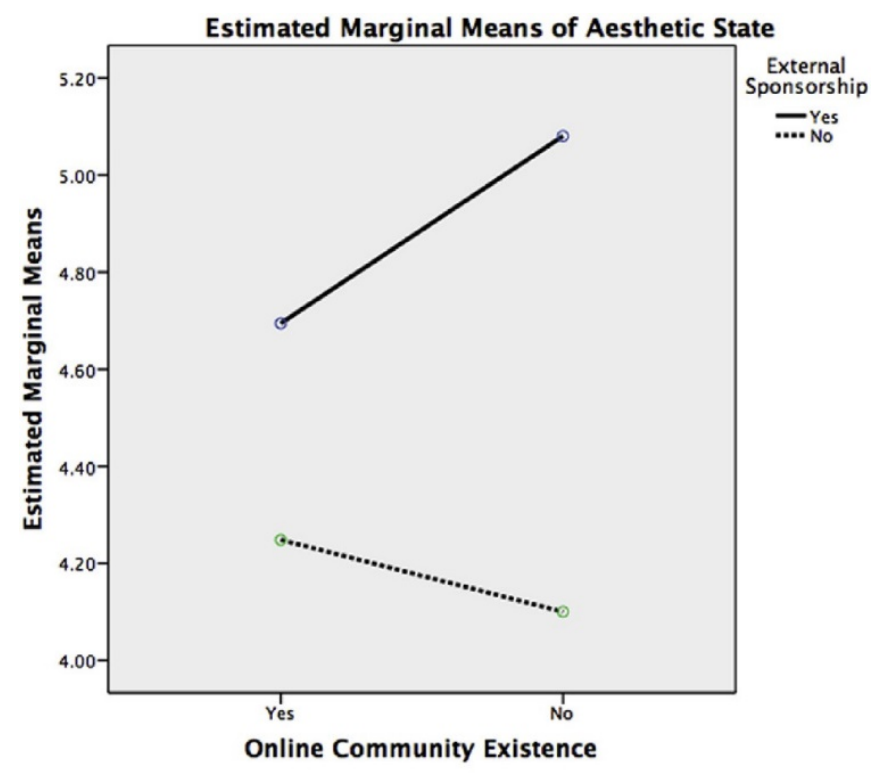

$F(1,186)=3.46, p=.05$.

Fig. 6. Interaction effect between existence of online community and external sponsorship on site aesthetic state. $F(1,186)=3.46, p=0.05$.

\section{Discussion}

Interfaces that encourage user contribution play a vital role in sustaining online communities (Preece, 2001). Most of the research in community sustainability and under-contribution topics has been devoted to either identifying successful community factors combatting lurking or empirically testing technological affordances, ones that are theoretically driven to serve as chief features for the community involvement and discourage lurking tendencies among community users (Farzan et al., 2011; Kim \& Sundar, 2014; Lampe et al., 2010; Ling et al., 2005; Preece et al., 2004; Ridings \& Gefen, 2004). However, the present study fundamentally questioned how likely real online communities - especially those dedicated to health - were equipped with adequate levels of the technological affordances as the literature has consistently and constantly suggested (e.g., Chidambaram \& Tung, 2005; Farzan et al., 2011; Kim \& Sundar, 2011; Ling et al., 2005). With this in mind, in order to translate an exploratory study into reality, the present study first reviewed two primary requirement aspects for thriving online health communities, namely the functional and structural.

\section{Discrepancy between real world situations and academic suggestions}

A content analysis with an exhaustive list of coded variables garnered from previous literature revealed the current state of user environments in online health communities (the features present to catalyst user contribution) was not aligned with what literature has been suggesting. Simple but critical information 
about community size was not available in most of the sites and online communities. Specifically, interface cues that signal structural aspects of online health communities were more poorly embedded than with general health websites. A very small number of online community sites were designed with basic interactive features for user-centered and user-to-user interactions. Perceived information accessibility was also lower among online health communities than the sites without the space for online health information sharing. Such poor structural cues found in these online health communities would not please users and thereby, naturally, would cease their life cycle (Chu, 2009; Ren \& Kraut, 2014).

While existing health websites and online health communities received an unsatisfactory grade on cuebased evaluations, a promising side was found in the present study for these communities functioning in their real-world context. The majority of health websites and online health communities offered not only indepth but also a wide range of health information, which might satisfy current and prospective users' resource expectations (Fig. 3). In terms of indicating the up-to-date state of community resources, a number of online health communities also presented more current, up-to-date information than the sites without any online community space. However, given the result from the focal health topic variables, online health communities did not seem as utilized by users who have great interest in serious and high-maintenance diseases compared to general health websites without any online community space (Fig. 1).

One might argue that online health communities need to focus on general, everyday health issues more than specialized health concerns. This is due to the limited capability of filtering valuable and safe health information that is ideally provided by experts (Sundar, Rice, Kim, \& Sciamanna, 2011). In this sense, a large volume of resource would be more important to community sustainability than foci of health concerns. Thus, with respect to functional cues mostly catering to online community users' resource needs, the real environment of online health communities may not be as seriously dysfunctional as previous literature suggests, capable of surviving in the current state.

\section{Rethinking the potential of external funding sources}

Nonetheless, the structural aspects of the analyzed online health communities in the present study did not seem successful to fit the requirements necessary for community success and sustainability. In fact, the infrastructure that facilitates interactivity, navigability and visually appealing layouts may need financial sources in order to construct a site with satisfactory interfaces and relevant technological affordances. Commercialized sites dedicated to knowledge sharing may not have this funding deficit problem and can maintain optimal online environments for user contribution (e.g., Yelp). This might hold true to other commercial sites as well, which, even in part, operate by electronic word-of-mouth (e.g., Amazon. com) as a similar concept of user contribution (Kim \& Sundar, 2014).

In fact, the present study found that external funding sources (i.e., advertisements and sponsorship) were fairly limited in health websites. Those sites without online communities hosted even less advertisements than those with them. Interestingly, the advertisements appeared to impede information accessibility. Such results are seemingly aligned to say that sites without online communities afford immediate access to information compared to than those with online communities. On the other hand, sponsorship assisted the sites to not only help users navigate better but also attract users with appealing interface designs. In particular, the relationship between sponsorship presence and the aesthetic states of interfaces was more pronounced in the sites without online communities than with them.

In other words, external sponsorship might be critical to create a healthy environment for user contribution by optimizing the capacity of structural tools, while advertisements could be left out in order to expedite the speed of information access, even at a perceptual level. Moreover, sponsorship may not seriously hurt the positive impressions of quality health information shared in the sites among users, either with or without online communities, as the users would meticulously examine the information. Hong (2006) pointed out that structural features of websites, advertisement and potential external funding would not significantly influence user's 
impression on the sites (i.e., perceived credibility). Unlike other websites (Flanagin \& Metzger, 2000), healthspecific domains draw visitors who are looking for specific information rather than merely wandering around for casual browsing (Hong, 2006).

Furthermore, this external sponsorship may play a primary role in helping sites and their online communities improve functional aspects, ultimately building better environments for user contribution in addition to bolstering more appealing structural aspects. Although privacy protection cues were found more in online health communities than the sites without such social support sharing possibility, the number of such cues was significantly limited (Fig. 5). Furthermore, while content moderation features were available more in online health communities than general health websites, the percentage of the online communities that offered users this feature did not outweigh that of the communities that did not contain it (Fig. 4). Financial sources could certainly mitigate the issues with these cues, signaling a more dependable online environment with richer resources.

\section{Limitations and future research}

Study limitations need to be addressed, especially for future research directions. First, due to its methodological nature, the present study cannot make claims that its reported findings account for the large, underlying reasons people still do not to contribute in online communities. However, the findings can suggest what people apparently see in these real-world environments when they seek health information online. In this sense, the current study still deems significant, practical implications to the field by employing a research method to conform to the primary purpose of research. For future research, a crosscheck-user survey might help add validity to the findings from the present study.

Second, generalizing the findings and their practical implications could be limited to health information sharing, which may not always be the case for other kinds of information and knowledge sharing. However, the purpose of this present study initially aimed to examine a peer-to-peer health communication context, since this area of online-information sharing appeared to suffer most from the problem of under-contribution (Fox \& Jones, 2009; Kim \& Sundar, 2011). Comparative, analytic research between successful and unsuccessful online communities, regardless of the information topics shared or the displayed support types, could underscore the findings from the present study.

In conclusion, it is apparent that the rapid development of communication technology expedited the revolution of online communities, creating a setting for health information and social support groups (Fox, 2011). However, the present study implies that the reality of health-information sharing in online environments does not quite utilize the advantages of this technological advancement. On one hand, the amount of information and social support shared in online health communication environments could have room to grow with such a wide range of health topics to be covered, largely intensive in nature. However, other indispensable elements of online environments for community sustainability - such as features that organize content, resources, interactivity, and other interface-cue related technology - appear far below mediocre regarding the environment's functional and structural matters. Future close, collaborative efforts need to be made among academic, health communication practitioners and these communities' actual web designers. 


\section{Appendix}

\begin{tabular}{|c|c|c|c|}
\hline $\begin{array}{l}\text { Variable } \\
\text { (Functional } \\
\text { aspects) }\end{array}$ & $\begin{array}{l}\text { Krippendorff's } \\
\text { alpha }\end{array}$ & $\begin{array}{l}\text { Variable } \\
\text { (Structural aspects) }\end{array}$ & $\begin{array}{l}\text { Krippendorff's } \\
\text { alpha }\end{array}$ \\
\hline $\begin{array}{l}\text { Community } \\
\text { existence }\end{array}$ & 0.79 & User profile & 0.99 \\
\hline Interest of a site & 0.73 & Membership history & 0.99 \\
\hline Topic breadth & 0.73 & $\begin{array}{l}\text { Membership contribution } \\
\text { history }\end{array}$ & 0.92 \\
\hline Topic depth & 0.89 & Member expertise & 0.77 \\
\hline $\begin{array}{l}\text { Topic filtering } \\
\text { System }\end{array}$ & 0.99 & Reply function & 0.87 \\
\hline $\begin{array}{l}\text { Content } \\
\text { moderation }\end{array}$ & 0.74 & Content evaluation & 0.99 \\
\hline $\begin{array}{l}\text { Information } \\
\text { currency }^{*}\end{array}$ & 0.84 & Content sharing & 0.86 \\
\hline \multirow{8}{*}{$\begin{array}{l}\text { Type of social } \\
\text { support }\end{array}$} & 0.99 & Buddy system & 0.99 \\
\hline & & Aesthetic state ${ }^{*}$ & 0.79 \\
\hline & & Accessibility $^{*}$ & 0.71 \\
\hline & & Login requirement & 0.94 \\
\hline & & Posting entry step ${ }^{*}$ & 0.99 \\
\hline & & Privacy protection & 0.94 \\
\hline & & Advertisements & 0.94 \\
\hline & & Sponsorship & 0.90 \\
\hline
\end{tabular}

Note. ${ }^{*}$ indicates an interval variable; otherwise nominal variable. 


\section{References}

Agaku, I. T., Adisa, A. O., Ayo-Yusuf, O. A., \& Connolly, G. N. (2014). Concern about security and privacy, and perceived control over collection and use of health information are related to withholding of health information from healthcare providers. Journal of the American Medical Informatics Association, 21,374-378.

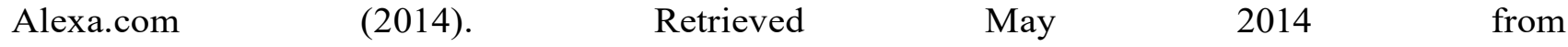
http://www.alexa.com/topsites/category/Top/Health/Support_Groups.

Banna, S., Hasan, H., \& Meloche, J. (2009). Opportunities for interactivity in public health websites: a content analysis approach. European and Mediterranean Conference on Information Systems, $1-14$.

Bateman, P. J., Gray, P. H., \& Butler, B. S. (2011). The impact of community commitment on participation in online communities. Information Systems Research, 22, 841-854. http://dx.doi.org/10.1287/isre.1090.0265.

Berlanga, A., Rusman, E., Bitter-Rijpkema, M., \& Sloep, P. (2009). Guidelines to foster interaction in online communities. In Learning network services for professional development (pp. 27-42). Springer Berlin Heidelberg.

Bishop, J. (2007). Increasing participation in online communities: a framework for human-computer interaction. Computers in Human Behavior, 23, 1881-1893. http://dx.doi.org/10.1016/j.chb.2005.11.004.

Butler, B. (2001). Membership size, communication activity, and sustainability: a resource-based model of online social structures. Information Systems Research, 12(4), 346-362.

Chaiken, S. (1987). The heuristic model of persuasion. In M. P. Zanna, J. M. Olson, \& C. P. Herman (Eds.), Social influence: The Ontario symposium (Vol. 5, pp. 3-39). Hillsdale, NJ: Erlbaum.

Chen, A. T. (2012). Exploring online support spaces: using cluster analysis to examine breast cancer, diabetes, and fibromyalgia support groups. Parent Education and Counseling, 87, 250-257.

Cheung, C., Lee, M., \& Lee, Z. (2013). Understanding the continuance intention of knowledge sharing in online communities of practice through the post-knowledge-sharing evaluation processes. Journal of the American Society for Information Science and Technology, 67, 13571374.

Chidambaram, L., \& Tung, L. L. (2005). Is out of sight, out of mind? an empirical study of social loafing in technology-supported groups. Information Systems Research, 16(2), 149-168. http://dx.doi.org/10.1287/isre.1050.0051.

Chu, K. M. (2009). A study of members' helping behavior in online community. Internet Research, 19, 279-292.

Chung, J. E., Park, N., Wang, H., Fulk, J., \& McLaughlin, M. (2010). Age differences in perceptions of online community participation among non-users: an extension of the technology acceptance model. Computers in Human Behavior, 26, 1674-1684.

Cline, R. J. W., \& Haynes, K. M. (2001). Consumer health information seeking on the Internet: the state of art. Health Education Research, 16, 671-692. http:// dx.doi.org/10.1093/her/16.6.671.

Connolly, T., \& Thorn, B. K. (1990). Discretionary databases: theory, data, and implications. In J. Fulk, \& A. Steinfield (Eds.), Organizations and communication technology (pp. 219-233). Sage Publications.

Coulson, N. S., Buchanan, H., \& Aubeeluck, A. (2007). Social support in cyberspace: a content analysis of communication within a Huntington's disease online support group. Patient Education and Counseling, 68, 173-178.

Cutrona, C. (1990). Stress and social support: in search of optimal matching. Journal of Social Clinical Psychology, 9(1), 3-14.

Du, Y. (2006). Modeling the behavior of lurkers in online communities using intentional agents. In Proceedings of the international conference on computational intelligence for modeling control and automation and international conference on intelligent agents web technologies and international commerce (CIMCA-IAWTIC'06) (pp. 60-66). 
Faraj, S., Jarvenpaa, S. L., \& Majchrzak, A. (2011). Knowledge creation in online communities. Organization Science, 22, 1224-1239.

Farzan, R., Dabbish, L., Kraut, R., \& Postmes, T. (2011). Increasing commitment to online communities by designing for social presence. In Proceedings of the 2011 annual conference on computer supported cooperative work (CSCW'11) (pp. 321-330).

Flanagin, A. J., \& Metzger, M. J. (2000). Perceptions of Internet information credibility. Journalism \& Mass Communication Quarterly, 77, 515-540.

Flanagin, A. J., \& Metzger, M. J. (2007). The role of site features, user attributes, and information verification behaviors on the perceived credibility of web-based information. New Media Society, 9, 319-342.

Flaviàn, C., Guinalíu, M., \& Gurrea, R. (2006). The role played by perceived usability, satisfaction and consumer trust on website loyalty. Information \& Management, 43, 1-14.

Fogg, B. J., Marshall, J., Laraki, O., Osipovich, A., Varma, C., Fang, N., ... Treinen, M. (2001, March). What makes web sites credible?: a report on a large quantitative study. In Proceedings of the SIGCHI conference on human factors in computing systems (pp. 61-68). ACM.

Fox, S. (2011). The social life of health information. Pew Internet \& American Life Project. Retrieved from http://pewinternet.org/Reports/2011/Social-Life-of- Health-Info.aspx.

Fox, S., \& Jones, S. (2009). The social life of health information. http://www. pewinternet.org/Reports/2009/8-The-Social-Life-of-Health-Information.aspx.

Ganley, D., \& Lampe, C. (2009). The ties that bind: social network principles in online communities. Decision Support System, 47, 266-274.

Heeter, C. (1989). Implications of new interactive technologies for conceptualizing communication. In Media use in the information age: Emerging patterns of adoption and consumer use (pp. 217-235).

Herndon, K. P., van Dam, A., \& Gleicher, M. (1994). The challenges of 3D interaction. ACM SIGCHI Bulletin, 26(4), 36-43.

Hong, T. (2006). The influence of structural and message features on web site credibility. Journal of the American Society of Information Science and Technology, 57(1), 114-127.

Jin, B., Park, J. Y., \& Kim, H. (2010). What makes online community members commit? a social exchange perspective. Behaviour \& Information Technology, 29, 587-599.

Jul, S. (2004). From brains to branch points: Cognitive constraints in navigational design. Unpublished doctoral dissertation. Ann Arbor: University of Michigan.

Kang, I., Lee, K. C., Lee, S., \& Choi, J. (2007). Investigation of online community volunteer behavior using cognitive map. Computers in Human Behavior, 23, 111-116.

Karau, S. J., \& Williams, K. D. (1995). Social loafing: research findings, implications, and future directions. Current Directions of Psychological Science, 4(5), 134-139.

Kim, H. S., \& Sundar, S. S. (2011). Using interface cues in online health community boards to change impressions and encourage user contribution. In Proceedings of the 2011 annual conference on human factors in computing systems (CHI' 11) (pp. 599-608).

Kim, H. S., \& Sundar, S. S. (2014). Can online buddies and bandwagon cues enhance user participation in online health communities? Computers in Human Behavior, 37, 319-333.

Kollock, P. (1999). The economies of online cooperation: gifts and public goods in cyberspace. In M. A. Smith, \& P. Kollock (Eds.), Communities in cyberspace (pp. 220-239). Routledge.

Kollock, P., \& Smith, P. (1999). Communities in cyberspace. London: Routledge.

Lampe, C., Wash, R., Velasquez, A., \& Ozkaya, E. (2010). Motivations to participate in online communities. In Proceedings of the 2010 annual conference on human factors in computing systems (CHI' 10) (pp. 1927-1936).

Latané, B. (1981). The psychology of social impact. American Psychologist, 36, 343-356.

Li, X. (2011). Factors influencing the willingness to contribute information to online communities. New Media \& Society, 13, 279-296.

Ling, K., Beenen, G., Ludford, P., Wang, X., Chang, K., \& Li, X. (2005). Using social psychology to motivate contributions to online communities. Journal of Computer-Mediated 
Communication, 10(4). Article 10.

Mo, P. K. H., \& Coulson, N. S. (2010). Empowering processes in online support groups among people living with HIV/AIDS: a comparative analysis of 'lurkers' and 'posters'. Computer in Human Behavior, 26, 1183-1193. http://dx.doi.org/10.1016/ j.chb.2010.03.028.

Moshagen, M., \& Thielsch, M. (2013). A short version of the visual aesthetics of websites inventory. Behavior \& Information Technology, 32, 1305-1311.

Nielsen, J. (2003). Usability 101. Retrieved from http://www.useit.com/alertbox/ 20030825.html.

Nonnecke, B., \& Preece, J. (1999). Shedding light on lurkers in online communities. In K. Buckner (Ed.), Ethnographic studies in real and virtual environments: Inhabited information spaces and connected communities (pp. 123-128). Edinburgh: Queen Margaret College.

Nonnecke, B., Preece, J., \& Andrews, D. (2004). What lurkers and posters think of each other. In Proceedings of the proceedings of the 37th annual Hawaii international conference on system sciences (HICSS'04). Track 7.

Oliver, P. E., \& Marwell, G. (1988). The paradox of group size in collective action: a theory of the critical mass. II. American Sociological Review, 53(1), 1-8.

Oliver, P. E., \& Marwell, G. (2001). Whatever happened to critical mass theory? a retrospective and assessment. Sociological Theory, 19(3), 292-311.

Petty, R. E., \& Cacioppo, J. T. (1986). Communication and persuasion: Central and peripheral routes to attitude change. New York: Springer Verlag.

Phang, C. W., Kankanhalli, A., \& Sabherwal, R. (2009). Usability and sociability in online communities: a comparative study of knowledge seeking and contribution. Journal of the Association for Information Systems, 10, 721-747.

Preece, J. (2000). Online communities: Designing usability, supporting sociability. Manchester, England: John Wiley \& Sons.

Preece, J. (2001). Sociability and usability: twenty years of chatting online. Behavior and Information Technology Journal, 20(5), 347-356.

Preece, J., \& Maloney-Krichmar, D. (2005). Online communities: design, theory, and practice. Journal of Computer-Mediated Communication, 10(4). article 1.

Preece, J., Nonnecke, B., \& Andrews, D. (2004). The top five reasons for lurking: improving community experiences for everyone. Computers in Human Behavior, 20, 201-223.

Ren, Y., \& Kraut, R. E. (2014). Agent-based modeling to inform online community design: impact of topical breadth, message volume, and discussion moderation on member commitment contribution. Human-Computer Interaction, 29, 351-389.

Ridings, C. M., \& Gefen, D. (2004). Virtual community attraction: why people hang out online. Journal of Computer-Mediated Communication, 10(1). http://dx.doi.org/10.1111/j.10836101.2004.tb00229.x.

Ridings, C., Gefen, D., \& Arinze, B. (2002). Some antecedents and effects of trust in virtual communities. Journal of Strategic Information Systems, 11(3e4), 271-295.

Stoerger, S. (2007). I'm not a doctor, but I play one on the web: credibility, funding, and interactivity features on health organization websites. Proceedings of the American Society for Information Science and Technology, 44(1), 1-5.

Sundar, S. S. (2008). The MAIN model: a heuristic approach to understanding technology effects on credibility. In M. J. Metzger, \& A. J. Flanagin (Eds.), Digital media, youth, and credibility (pp. $72-$ 100). Cambridge, MA: The MIT Press.

Sundar, S. S., Bellur, S., Oh, J., Xu, Q., \& Jia, H. (2014). User experience of on-screen interaction techniques: an experimental investigation of clicking, sliding, zooming, hovering, dragging and flipping. Human Computer Interaction, 29, 109-152.

Sundar, S. S., Oh, J., Bellur, S., Jia, H., \& Kim, H. S. (2012). Interactivity as self-expression: a field experiment with customization and blogging. In Proceedings of the 2012 annual conference on human factors in computing systems (CHI'12) (pp. 395-404).

Sundar, S. S., Rice, R. E., Kim, H. S., \& Sciamanna, C. (2011). Online health information: conceptual 
challenges and theoretical opportunities. In T. Thompson,

R. Parrott, \& J. Nussbaum (Eds.), Handbook of health communication (2nd ed., pp. 181-202). New York: Routledge.

Thielsch, M. T., Blotenberg, I., \& Jaron, R. (2013). User evaluation of websites: from first impression to recommendation. Interacting with Computers. iwt033.

Toral, S. L., Martinez-Torres, M. R., Barrero, F., \& Cortés, F. (2009). An empirical study of the driving forces behind online communities. Internet Research., 19, 378-392.

Turner, K. W., Grube, J. A., \& Meyers, J. (2001). Developing an optimal match within online communities: an exploration of CMC support communities and traditional support. Journal of Communication, 51, 231-252.

Valacich, J. S., Dennis, A. R., \& Nunamaker, J. F. (1992). Group size and anonymity effects on computermediated idea generation. Small Group Research, 23(1), 49-73. http://dx.doi.org/10.1177/1046496492231004.

Voelpel, S. C., Eckhoff, R. A., \& Förster, J. (2012). David against Goliath? group size and bystander effects in virtual knowledge sharing. Human Relations, 61, 271-295.

Wang, X., \& Clay, P. F. (2012). Beyond adoption intention: online communities and member motivation to contribute longitudinally. Journal of Organizational Computing and Electronic Commerce, 23, 215-236.

Wasko, M. M., \& Faraj, S. (2000). "It is what one does": why people participate and help others in electronic communities of practice. Journal of Strategic Information Systems, 9(2-3), 155-173. http://dx.doi.org/10.11016/s0963-8687(00) 00045-7.

Wright, K. B., \& Bell, S. B. (2003). Health-related support groups on the Internet: linking empirical findings to social support and computer-mediated communication theory. Journal of Health Psychology, 8, 39-54.

Wright, K. B., Johnson, A. J., Averbeck, J., \& Bernard, D. (2011). Computer-mediated social support groups. In T. L. Thompson, R. Parrott, \& J. F. Nussbaum (Eds.), Routledge handbook of health communication (2nd ed.). Thousand Oaks, CA: Routledge. Sage.

Yeager, J. (2011). A content analysis of an online support group for survivors of sexual violence. In E-health communities and online self-help groups: Applications and usage (pp. 85-106). Hershey, PA: IGI Global.

Zajonc, R. B. (1965). Social facilitation. Science, 149(3681), 269-274.

Zajonc, R. B., \& Brickman, P. (1969). Expectancy and feedback as independent factors in task performance. Journal of Personality and Social Psychology, 11, 148-156.

Zhang, Q., \& Yang, Y. (2009, November). A study of positive effects on user experience in navigation. In Computer-aided industrial design \& conceptual design, 2009. CAID \& CD 2009. IEEE 10th international conference on (pp. 444-447). IEEE.

Further reading

Cutrona, C., Russell, D., \& Rose, J. (1986). Social support and adaptation to stress by the elderly. Journal of Psychology and Aging, 1(1), 47-54. 